\title{
S-1 in Patients with Advanced Esophagogastric Adenocarcinoma: Results from the Safety Compliance Observatory on Oral fluoroPyrimidines (SCOOP) Study
}

\author{
Marino Venerito ${ }^{1}$ (D) \\ Published online: 1 March 2019 \\ (c) The Author(s) 2019
}

\begin{abstract}
Background and Objectives S-1-based regimens have been shown to be as effective as other fluoropyrimidine-based regimens with a better safety profile in patients with advanced esophagogastric adenocarcinoma. However, real-world data on S-1 in European patients with advanced esophagogastric adenocarcinoma are lacking. The Safety Compliance Observatory on Oral fluoroPyrimidines (SCOOP) study evaluated safety and relative dose intensities for patients treated with S-1-based regimens for advanced esophagogastric adenocarcinoma as part of daily practice.

Methods Overall, data for 125 patients with advanced esophagogastric adenocarcinoma were collected at 21 centers in five countries in Europe. Demographics, treatment, and adverse-event data were recorded over a planned treatment of six cycles. Results Most patients (87\%) received combination treatment of S-1 plus a platinum compound. Adverse events related to S-1 treatment were mostly grade 1 or 2 while reported grade 3-4 serious adverse events related to S-1 occurred in 12 patients and were most often grade 3 neutropenia $(n=4,3.2 \%)$ or diarrhea $(n=5,4 \%)$. The most common adverse events of any grade that were attributable to S-1 treatment included neutropenia, anemia, thrombocytopenia, diarrhea, nausea, vomiting, and fatigue. No patients experienced mucositis, dehydration, or febrile neutropenia, whereas $2 \%$ (3/125) of patients experienced hand-foot syndrome.

Conclusion The overall relative dose intensity was $70 \%$. In a real-world setting, patients with advanced esophagogastric adenocarcinoma tolerated S-1 treatment well with high compliance rates. The SCOOP study provides valuable information on S-1 relative dose intensity that can be used for treatment decision making.
\end{abstract}

\section{Key Points}

The Safety Compliance Observatory on Oral fluoroPyrimidines (SCOOP) provides real-world data on adverse events and relative dose intensity of S-1 therapy in patients with advanced esophagogastric adenocarcinoma

Adverse events related to $\mathrm{S}-1$ treatment were mostly grade 1 or 2 while reported grade 3-4 serious adverse events related to $\mathrm{S}-1$ occurred in 12 patients and were most often grade 3 neutropenia $(n=4,3.2 \%)$ or diarrhea $(n=5,4 \%)$. Notably, only $2 \%$ (3/125) of patients experienced hand-foot syndrome

The overall relative dose intensity was $70 \%$. This information can be used for treatment decision making

Marino Venerito

m.venerito@med.ovgu.de

Otto-von-Guericke University Hospital, Department of Gastroenterology, Hepatology and Infectious Diseases, Leipziger Str. 44, 39120 Magdeburg, Germany

\section{Introduction}

Esophagogastric adenocarcinoma, including adenocarcinoma of the gastroesophageal junction and stomach, is a major health concern [1]. In approximately two-thirds of cases, esophagogastric adenocarcinoma is diagnosed at an advanced stage, with regional lymph node invasion or distant metastases [2]. In cases with advanced T stage and/ or regional lymph node involvement (T3/4 or $\mathrm{N}+$ ), but no evidence of distant metastases, perioperative chemotherapy significantly improves the survival of patients undergoing surgical resection compared with surgery alone [3, 4]. For patients with metastatic disease, palliative chemotheapy improves survival compared with best supportive care alone [5]. Recommended treatment for patients with esophagogastric adenocarcinoma and advanced disease is generally a fluoropyrimidine-platinum doublet [5-fluorouracil (5-FU) or capecitabine plus cisplatin or oxaliplatin] or triplet (addition of epirubicin or docetaxel) to the fluoropyrimidine-platinum regimen [5, 6]. In addition, 
trastuzumab should be added to 5-FU/capecitabine plus cisplatin in HER-2-positive patients with metastatic disease $[5,6]$.

In 2011, a third fluoropyrimidine option, Teysuno ${ }^{\circledR}$, was approved for use in Europe in combination with cisplatin for patients with advanced esophagogastric adenocarcinoma [7]. Teysuno ${ }^{\circledR}$, also known worldwide under the International Nonproprietary Name S-1, is a combination of three drugs, tegafur (a 5-FU prodrug), gimeracil (a dihydropyrimidine dehydrogenase inhibitor), and oteracil (an orotate phosphoribosyl transferase inhibitor) in a formulation designed to provide effective plasma concentrations of 5-FU while reducing the well-known toxicities of 5-FU and capecitabine [e.g., hematological effects, gastrointestinal toxicity, and hand-foot syndrome (HFS)] $[8,9]$.

$\mathrm{S}-1$ is a fourth-generation oral fluoropyrimidine that has been approved in Asian countries for the treatment of advanced esophagogastric adenocarcinoma and is highly active at a dose of $40 \mathrm{mg} / \mathrm{m}^{2}$ twice daily when used in combination with cisplatin in Japanese patients with advanced esophagogastric adenocarcinoma with a reported overall response rate of $76 \%$ [10]. However, tolerability of S-1 differs between Asian patients and Western patients owing to polymorphisms in the CYP2A6 gene that result in differences in the manner in which Western patients metabolize the tegafur component [11]. Based on pharmacokinetic data, $\mathrm{S}-1$ is administered at a dose of $25 \mathrm{mg} /$ $\mathrm{m}^{2}$ twice daily for 21 days of a 28-day cycle when used in combination with a platinum salt (cisplatin) in Western patients $[7,11]$.

$\mathrm{S}-1$ is as effective as 5-FU and capecitabine, but has a better safety profile. In FLAGS (First-Line Advanced Gastric Cancer Study), the combination of S-1 with cisplatin was non-inferior to infusional fluorouracil with cisplatin in overall survival for patients with advanced gastric or gastroesophageal adenocarcinoma, but resulted in a significantly improved safety profile [8].

In a network analysis of clinical trials of 5-FU, capecitabine, and S-1 in previously untreated esophagogastric adenocarcinoma, patients receiving S- 1 had similar overall survival and progression-free survival compared to those receiving intravenous (IV) 5-FU or oral capecitabine [9]. Moreover, S-1 treatment was associated with lower rates of some important adverse events including fewer catheter-related complications, grade 3-4 mucositis, stomatitis, febrile neutropenia, dehydration, and toxicity-related deaths than 5-FU, and fewer cases of grade 3-4 neutropenia and grade 1-2 HFS compared with capecitabine [9]. Similarly, in a recent phase III study that compared the incidence of HFS in patients with metastatic colorectal cancer treated with oral capecitabine vs. S-1, efficacy was similar but rates of HFS were significantly lower for patients who received S-1 compared with capecitabine ( $45 \%$ vs. $73 \% ; p=0.0005$ ) [12].
S-1 has also been shown to be safe when used after intolerability of capecitabine. In a retrospective analysis of data for patients who received capecitabine as treatment for a solid tumor and discontinued because of HFS, $94 \%$ were able to reduce the grade of HFS or completely resolve their HFS after switching to S-1 [13]. In addition, in patients with capecitabine-related cardiotoxicity, which has been observed in $5.9 \%$ of patients and often results in treatment discontinuation, there is evidence that they can be safely switched to S-1 without recurrence of cardiac toxicity [14-16].

Thus, S-1 offers an equally effective and better tolerated fluoropyrimidine treatment alternative to capecitabine and IV 5-FU in patients with advanced esophagogastric adenocarcinoma for whom compliance and drug exposure are key to efficacy and safety against a rapidly advancing disease. To evaluate compliance and drug exposure rates in the daily practice of patients receiving S-1 for advanced esophagogastric adenocarcinoma, we conducted the Safety Compliance Observatory on Oral fluoroPyrimidines (SCOOP) study. Here, we report the real-world results from the analysis of safety and dose modification/dose intensity surveillance data for 125 Caucasian patients with advanced esophagogastric adenocarcinoma who received S-1 monotherapy or combination therapy for a planned six cycles of treatment.

\section{Materials and Methods}

SCOOP was an observational study conducted at 21 centers in five countries: Ireland, Greece, Italy, Germany, and Austria. Each center was asked to include at least five patients to reduce centric effects. The study was approved by the institutional review board and/or ethics committee at each participating center and was in accordance with the Helsinki Declaration of 1975, as revised in 1983.

The main objective of the study was to evaluate the relative dose intensity of S-1 formulations during up to six treatment cycles. Secondary objectives included evaluation of the proportion of patients undergoing six cycles of treatment without any dose reduction or delays in S-1 dosage and to record rates of adverse events observed in daily practice.

Patients were aged 18 years or older, had histologically confirmed Stage III b/c, Stage IV, or relapsed esophagogastric adenocarcinoma, had an Eastern Cooperative Oncology Group performance status of $\leq 2$, were able to take oral medications, had adequate organ function to receive chemotherapy, and provided written consent after receiving information about the trial. Patients with contraindications for oral fluoropyrimidine treatment and women of childbearing age who were not using effective contraception were excluded. 
Treatment was planned as a maximum of six cycles of an S-1-based regimen. All decisions regarding dosing, dose adjustment, discontinuation, and additional therapies were left to the discretion of the local oncologist. The relative dose intensity for patients over six cycles was calculated as the dose actually received over the planned dose based on the starting dose of S-1. Patients for whom data ended without explanation were assumed to be permanently discontinued and dose intensity was counted for each cycle that included data for the S-1 dose. Discontinuation of S-1 treatment for any reason led to withdrawal from the study.

Patients were monitored throughout the study and laboratory testing (hematology, liver function, renal function, serum electrolyte testing) was performed according to local practice. Owing to the observational nature of the study, there were no required visits or examinations but patients were asked to record doses taken and adverse events in a patient diary to be reviewed at each visit. Demographic data, treatment history, disease histology and characteristics, treatment regimen, adverse events, treatment modifications/discontinuations, and the relationship of adverse events to S-1 treatment were recorded in a database for the baseline visit, post-cycle, and end of follow-up visits. Adverse events were scored according to National Cancer Institute Common Terminology Criteria for Adverse Events, Version 3.0 [17].

This study was non-interventional in nature and only descriptive statistical analyses were performed. No efficacy evaluations were performed.

\section{Results}

\subsection{Patient Characteristics}

Data for 125 patients [Ireland $(n=25)$, Greece $(n=62)$, Austria $(n=1)$, Italy $(n=11)$, and Germany $(n=26)$ ] were recorded over six cycles. Patient characteristics are shown in Table 1. Patients were $66.4 \%$ male with a mean age of 64.5 years. Thirty-two percent of patients were aged older than 70 years. Most had Stage III b/c disease (66.4\%) and tumors located in the stomach $(59.2 \%)$ or gastroesophageal junction (29.6\%). Older patients (aged $\geq 70$ years) had higher rates of Stage IV disease (67.5\%). With respect to the Laurén classification [18], 56.8\% of study participants had diffuse adenocarcinoma histology. Histology data were not entered into the database for 31 patients (24.8\%).

The most common co-morbidities were cardiovascular disease ( $43.2 \%$ overall; $57.5 \%$ in patients aged $\geq 70$ years) and metabolic disorders (30.4\%). Most patients (87\%) started on combination therapy with a platinum compound [cisplatin $(57 \%)$, oxaliplatin $(31 \%)$, carboplatin (12\%)], whereas $13 \%$ received S-1 monotherapy. Ten patients $(8.0 \%)$ received triplet therapy: S-1 plus oxaliplatin with docetaxel $(n=1)$ or with epirubicin $(n=8)$, and S-1 plus carboplatin with epirubicin $(n=1)$. Less than $20 \%$ of patients had received previous therapy with capecitabine or IV 5-FU. Two patients had discontinued because of toxicity during IV 5-FU treatment.

\subsection{Adverse Events}

Recorded adverse events are shown in Table 2. The most common adverse events were neutropenia, anemia, thrombocytopenia, diarrhea, nausea, and fatigue. There were no cases of mucositis, febrile neutropenia, or dehydration. A total of 83 patients $(66.4 \%)$ experienced an adverse event of any grade during treatment, 22 patients $(17.6 \%)$ experienced grade 3 or 4 adverse events.

Table 1 Patient characteristics

\begin{tabular}{lll}
\hline Characteristics & $N(\%)$ \\
\hline Male & $83(66.4)$ & \\
Median age, years (range) & $64(29-90)$ & \\
$70+$ & $40(32)$ & \\
Tumor status at enrollment & & \\
Stage III b/c & $83(66.4)$ & \\
Stage IV/relapsed & $42(33.6)$ & \\
Tumor site & & \\
Stomach & $74(59.2)$ & \\
GEJ & $37(29.6)$ & \\
Stomach and GEJ & $13(10.4)$ & \\
Not specified & $1(0.8)$ & \\
Histopathology & & \\
Diffuse/signet ring adenocarcinoma & $71(56.8)$ & \\
Intestinal adenocarcinoma & $19(15.2)$ & \\
Mixed & $4(3.2)$ & \\
Not reported & $31(24.8)$ & \\
Co-morbidities ${ }^{\mathrm{b}}$ & $\mathrm{All}$ & \\
Cardiovascular & $54(43.2)$ & $23(57.5)$ \\
Metabolic & $38(30.4)$ & $13(32.5)$ \\
Renal & $8(6.4)$ & $3(7.5)$ \\
Hepatic & $5(4.0)$ & \\
Pulmonary (COPD) & $4(3.2)$ & \\
Depression & $3(2.4)$ & \\
Previous capecitabine or IV 5-FU & $23(18)$ \\
Received S-1 plus platinum combination & $109(87)$ \\
t reatment in this study & & \\
\hline
\end{tabular}

$C O P D$ chronic obstructive pulmonary disease, GEJ gastroesophageal junction, IV 5-FU intravenous 5-fluorouracil

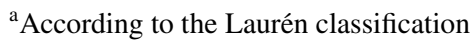

${ }^{b}$ Other co-morbidities reported that were present in two or fewer patients included psychosis syndrome, Sjogren syndrome, lung cancer, iron deficiency anemia, polymyalgia, and hepatitis B virus 


\subsubsection{S-1 Treatment}

Table 3 shows the relationship between adverse events and S-1 treatment. Data for adverse events reported over six cycles for the 125 patients who started treatment are shown. The most common adverse events attributable to S- 1 treatment included neutropenia, anemia, thrombocytopenia, diarrhea, nausea, vomiting, and fatigue. Thirty patients (24\%) experienced additional adverse events classified as "other" in the database, $13(10.4 \%)$ of these were considered to be related to S-1 treatment. Of these other adverse events, leukopenia, anorexia, dry mouth, dyspnea, and dysgeusia were considered related to $\mathrm{S}-1$ treatment. Although cardiac co-morbidities were common in this cohort of patients ( $43.2 \%$ overall and $57.5 \%$ among those aged over 70 years), only one patient (a 56-year-old man with pre-existing cardiovascular disease) in the study experienced a cardiovascular adverse event (grade 1 chest pain) and that was determined to be unrelated to S-1 treatment. Three patients (2.4\%) experienced HFS at some point during S-1 treatment, two had grade 1 HFS and one had grade 3 HFS in cycles two and three that resolved with $\mathrm{S}-1$ dose reduction.

\subsubsection{Platinum Treatment}

Of the 22 patients who experienced grade $3 / 4$ adverse events, $18(82 \%)$ received platinum combination therapy.
Data were not collected on whether specific adverse events were attributable to platinum compounds.

\subsubsection{Previous 5-Fluorouracil Treatment}

Of the 23 patients who received previous treatment with IV 5-FU or capecitabine, 15 (65\%) experienced adverse events, six (26\%) of them grade 3 or 4 , during S-1 treatment including grade 3 neutropenia, thrombocytopenia, diarrhea, pain, and hearing loss and one case of grade 4 infection (pneumonia). Among patients with previous exposure to 5-FU regimens, no HFS was observed during the six cycles of S-1 treatment. One patient experienced grade 2 diarrhea in cycle two and two patients experienced grade 3 diarrhea in cycle six that was judged to be related to $\mathrm{S}-1$ treatment.

\subsection{Dose Modifications/Discontinuations}

Overall, 54 (43\%) patients completed the study. Eight patients died during the study and none of these deaths was attributed to S-1 treatment. There were 46 dose modifications. Furthermore, 47 out of 125 patients (38\%) permanently discontinued the intake of S-1 because of progressive disease $(n=32,26 \%)$, adverse events $(n=14$,

Table 2 Adverse events by cycle

\begin{tabular}{|c|c|c|c|c|c|c|c|c|c|c|c|c|}
\hline \multirow[t]{2}{*}{$\begin{array}{l}\text { Grade } \\
n(\%)\end{array}$} & \multicolumn{2}{|l|}{$\begin{array}{l}\text { Cycle } 1 \\
n=125\end{array}$} & \multicolumn{2}{|l|}{$\begin{array}{l}\text { Cycle } 2 \\
n=118\end{array}$} & \multicolumn{2}{|l|}{$\begin{array}{l}\text { Cycle } 3 \\
n=106\end{array}$} & \multicolumn{2}{|l|}{$\begin{array}{l}\text { Cycle } 4 \\
n=86\end{array}$} & \multicolumn{2}{|l|}{$\begin{array}{l}\text { Cycle } 5 \\
n=63\end{array}$} & \multicolumn{2}{|l|}{$\begin{array}{l}\text { Cycle } 6 \\
n=56\end{array}$} \\
\hline & All & $3 / 4$ & All & $3 / 4$ & All & $3 / 4$ & All & $3 / 4$ & All & $3 / 4$ & All & $3 / 4$ \\
\hline Neutropenia & $14(11.2)$ & $4(3.2)$ & $13(11.0)$ & $3(2.5)$ & $7(6.6)$ & $1(0.9)$ & $11(12.8)$ & 0 & $6(9.5)$ & $1(1.6)$ & $5(8.9)$ & $1(1.8)$ \\
\hline Febrile neutropenia & 0 & 0 & 0 & 0 & 0 & 0 & 0 & 0 & 0 & 0 & 0 & 0 \\
\hline Anemia & $38(30.4)$ & $1(0.8)$ & $29(24.6)$ & 0 & $24(22.6)$ & 0 & $24(27.9)$ & 0 & $15(23.8)$ & 0 & $12(21.4)$ & $1(1.8)$ \\
\hline Thrombocytopenia & $9(7.2)$ & $1(0.8)$ & $8(6.8)$ & $2(1.7)$ & $9(8.5)$ & 0 & $5(5.8)$ & 0 & $5(7.9)$ & 0 & $7(12.5)$ & $1(1.8)$ \\
\hline HFS & $1(0.8)$ & 0 & $1(0.8)$ & $1(0.8)$ & $1(0.9)$ & $1(0.9)$ & $1(1.2)$ & 0 & 0 & 0 & $1(1.8)$ & 0 \\
\hline Cardiac disorders & $1(0.8)$ & 0 & 0 & 0 & 0 & 0 & 0 & 0 & 0 & 0 & 0 & 0 \\
\hline Diarrhea & $12(9.6)$ & $1(0.8)$ & $8(6.8)$ & $2(1.7)$ & $2(1.9)$ & 0 & $1(1.2)$ & 0 & 0 & 0 & $3(5.6)$ & $3(5.6)$ \\
\hline Stomatitis & $1(0.8)$ & 0 & 0 & 0 & 0 & 0 & 0 & 0 & 0 & 0 & 0 & 0 \\
\hline Mucositis & 0 & 0 & 0 & 0 & 0 & 0 & 0 & 0 & 0 & 0 & 0 & 0 \\
\hline Nausea & $9(7.2)$ & 0 & $4(3.4)$ & 0 & $6(5.7)$ & 0 & $4(4.7)$ & 0 & $2(3.2)$ & 0 & $2(3.6)$ & 0 \\
\hline Vomiting & $4(3.2)$ & 0 & $2(1.7)$ & $1(0.8)$ & $2(1.9)$ & 0 & 0 & 0 & 0 & 0 & $2(3.6)$ & 0 \\
\hline Weight loss & $5(4.0)$ & 0 & $2(1.7)$ & 0 & 0 & 0 & 0 & 0 & 0 & 0 & 0 & 0 \\
\hline Skin rash & $1(0.8)$ & 0 & 0 & 0 & 0 & 0 & 0 & 0 & 0 & 0 & 0 & 0 \\
\hline Hearing loss & $1(0.8)$ & $1(0.8)$ & $1(0.8)$ & $1(0.8)$ & 0 & 0 & 0 & 0 & 0 & 0 & 0 & 0 \\
\hline Sensory neuropathy & $3(2.4)$ & 0 & $3(2.5)$ & 0 & 0 & 0 & 0 & 0 & $2(3.2)$ & 0 & $1(1.8)$ & 0 \\
\hline Fatigue & $11(8.8)$ & $1(0.8)$ & $12(10.2)$ & 0 & $7(6.6)$ & 0 & $3(3.5)$ & $1(1.2)$ & $4(6.3)$ & $1(1.6)$ & $7(12.5)$ & $1(1.8)$ \\
\hline Fever & 0 & 0 & 0 & 0 & $1(0.9)$ & 0 & 0 & 0 & 0 & 0 & $1(1.8)$ & 0 \\
\hline Infection & $1(0.8)$ & $1(0.8)$ & $2(1.7)$ & $2(1.7)$ & $2(1.9)$ & $2(1.9)$ & 0 & 0 & 0 & 0 & 0 & 0 \\
\hline Dehydration & 0 & 0 & 0 & 0 & 0 & 0 & 0 & 0 & 0 & 0 & 0 & 0 \\
\hline Dry eye syndrome & 0 & 0 & $1(0.8)$ & 0 & 0 & 0 & 0 & 0 & 0 & 0 & 0 & 0 \\
\hline
\end{tabular}

HFS hand foot syndrome 
Table 3 Adverse events (AE): relationship to $\mathrm{S}-1$ treatment

\begin{tabular}{llll}
\hline & $\begin{array}{l}\text { Number of patients who experi- } \\
\text { enced AE, } n(\%)\end{array}$ & $\begin{array}{l}\text { S-1 related, } \\
n(\%)\end{array}$ & $\begin{array}{l}\text { Grade 3/4 related } \\
\text { to S-1, } n(\%)\end{array}$ \\
\hline Neutropenia & $21(16.8)$ & $11(8.8)$ & $4(3.2)$ \\
Febrile neutropenia & 0 & 0 & 0 \\
Anemia & $48(38.4)$ & $11(8.8)$ & 0 \\
Thrombocytopenia & $20(16.0)$ & $8(6.4)$ & 0 \\
HFS & $3(2.4)$ & $3(2.4)$ & $1(0.8)$ \\
Cardiac disorders & $1(0.8)$ & 0 & 0 \\
Diarrhea & $18(14.4)$ & $11(8.8)$ & $5(4.0)$ \\
Stomatitis & $1(0.8)$ & $1(0.8)$ & 0 \\
Mucositis & 0 & 0 & 0 \\
Nausea & $15(12.0)$ & $13(10.4)$ & 0 \\
Vomiting & $6(4.8)$ & $4(3.2)$ & $1(0.8)$ \\
Weight loss & $6(4.8)$ & $1(0.8)$ & 0 \\
Skin rash & $1(0.8)$ & $1(0.8)$ & 0 \\
Hearing loss & $1(0.8)$ & 0 & 0 \\
Sensory neuropathy & $7(5.6)$ & $2(8.0)$ & 0 \\
Fatigue & $21(16.8)$ & $14(11.2)$ & $1(0.8)$ \\
Fever & $2(8.0)$ & 0 & 0 \\
Infection & $3(2.4)$ & 0 & 0 \\
Dehydration & 0 & 0 & 0 \\
Dry eye syndrome & $1(0.8)$ & 0 & 0 \\
Other & $30(24.0)$ & $13(10.4)^{\mathrm{b}}$ & \\
\hline
\end{tabular}

HFS hand foot syndrome

aAdverse events classified as "other" in the database included burning eyelids, sleep disorders, dizziness, constipation, leukopenia, anorexia, edema, hematoma, pain, hair loss, dysgeusia, dysosmia, hypokalemia, hyponatremia, thrombosis, dyspnea, gastroesophageal reflux, dry mouth, bilirubin increase, hyperuricemia, hyperkalemia, asthenia, and peripheral paresthesias

bAdverse events classified as "other" in the database and attributed to S-1 treatment included leukopenia, anorexia, dry mouth, dyspnea, and dysgeusia
$11 \%)$, or non-compliance $(n=1,0.8 \%)$. Follow-up data were not complete for an additional 16 patients (13\%).

\subsection{Relative Dose Intensity}

The average relative dose intensity for 125 patients who received S-1 for at least one cycle was $69.8 \%$. Forty-eight patients $(38 \%)$ received $100 \%$ of the planned dose and an additional 13 patients (10\%) received more than $80 \%$ of the planned dose.

\subsubsection{Relative Dose Intensity and Adverse Events}

The relative dose intensity for the 22 patients who experienced grade $3 / 4$ adverse events was $67 \%$. For all patients who experienced grade $3 / 4$ adverse events, the reported initial dose of S-1 was higher than $17 \mathrm{mg} / \mathrm{m}^{2}$ twice daily.

\section{Discussion}

The present study provides valuable real-life data about the relative dose intensity and safety of S-1-based treatments for European patients with advanced esophagogastric cancer. Relative dose intensity data for S-1 have not been reported so far, and complement evidence on S-1 acquired in the FLAGS trial is highly desirable [8]. Patient selection criteria were similar between FLAGS and SCOOP to allow comparisons [8]. The relative dose intensity for S- 1 treatment in SCOOP was $69.8 \%$, suggesting that S-1 is well tolerated. Overall, $38 \%$ of patients received $100 \%$ of the planned S-1 dose (mostly as part of a regimen with other cytostatic drugs). Dose intensities and adverse-event rates may differ among subgroups of patients according to disease stage, therapy goal (i.e., neoadjuvant vs. palliative), and regimen used (i.e., doublet vs. triplet). The limited 
number of recruited patients prevented subgroup analyses for addressing these issues. Information on relative dose intensity is of value for treatment decision making in modern oncology. Especially in elderly patients, a drug may still be effective even if the starting dose is reduced or a dose reduction is required $[19,20]$.

Adverse events observed in SCOOP did not differ from those reported in FLAGS. Anemia, neutropenia, thrombocytopenia, nausea, and vomiting were relatively common with S-1 treatment in both studies whereas febrile neutropenia, mucositis, stomatitis, and dehydration rates were very low or did not occur [8]. Moreover, HFS was only experienced by roughly $2 \%$ of SCOOP patients, whereas the rate of HFS observed in a real-world setting among patients receiving capecitabine monotherapy for metastatic colorectal cancer was as high as $47 \%$ [21].

Discontinuation rates observed in $\mathrm{SCOOP}$ as a result of progressive disease $(26 \%)$ were similar to those reported for patients with metastatic colorectal cancer taking capecitabine monotherapy in the above-mentioned realworld survey (up to 24\%) but roughly half of what was observed in FLAGS (62\%). While this might be a reflection of the real-world setting $[8,21]$, a more plausible explanation for this observation is the use of S-1-based chemotherapy in a perioperative setting in which only four cycles (two each in the neoadjuvant and adjuvant settings) are foreseen [22]. The latter explanation is supported by the fact that the majority of patients included in SCOOP (66\%) had locally advanced disease without distant metastasis, a clear indication for perioperative chemotherapy according to the current guidelines [6, 23, 24]. Although this hypothesis was not systematically verified in every participating center, a single-center retrospective review of the SCOOP data from Magdeburg, Germany showed that five out of 14 patients (36\%) recruited for SCOOP received S-1-based chemotherapy in a perioperative setting. Thus, the lower discontinuation rates as a result of progressive disease associated with $\mathrm{S}-1$ administration in SCOOP compared with FLAGS are more likely related to the therapy setting in which S-1 was employed (no more than four cycles required) rather than to the tolerability of the drug.

One aspect of this study that differs from that of preapproval clinical trials of the use of S-1 is the use of off-label combinations of S-1 with platinum compounds. S-1 is currently approved in advanced esophagogastric adenocarcinoma in combination with cisplatin [7]. However, physicians in this study reported the use of S-1 in off-label combinations with oxaliplatin and carboplatin. These off-label uses were clustered by country and site. For example, carboplatin was administered to two patients at a single center in Germany, while all other German centers used cisplatin. Similarly, a single center in Greece administered carboplatin to
11 patients and oxaliplatin to ten patients but cisplatin to only one patient while other Greek centers used only cisplatin. In Ireland, the single included center used oxaliplatin in 24 patients and cisplatin in four patients when combination treatment was prescribed. These off-label uses appear to reflect local preferences.

Carboplatin, in particular, does not appear in guidelines for gastric cancer therapy. However, some oncologists may prefer carboplatin to cisplatin based on trials in other cancers (ovarian and lung cancer) because in cisplatin-based therapies there is the need for prolonged infusion times for hydration, but this is not the case for carboplatin. Some oncologists may prefer oxaliplatin to cisplatin because cisplatin induces more vomiting, but this aspect has been overcome with modern antiemetic drugs [25].

This study was limited by some real-world aspects of data collection at the various treatment sites. For example, an unusually high number of patients in this study (10.4\%) were listed as having both stomach and gastroesophageal junction disease. This is an artifact of the design of the data entry worksheet that clearly highlights the difficulty of correctly distinguishing and classifying gastric tumors invading the gastroesophageal junction from junctional cancers infiltrating large areas of the stomach. In addition, histology data were not entered into the database for $24 \%$ of patients. The notion that histology was missing in $24 \%$ of patients is implausible and we believe this is also a reflection of the real-world pressures of additional record keeping in busy daily practice. For some patients, the data were not complete and it is unclear whether the patients discontinued or were simply not followed up in the data set. The percentages for discontinuations and dose modifications reflect the percentage of patients for whom data were available for each cycle.

The database was tested for plausibility and for cases with clear implausibility, data were corrected. For example, two patients with HFS grade 1 and 3 were listed as having adverse events not related to S-1 treatment. This was clearly recognized as a data entry error and corrected. Indeed, one of the patients was only receiving S-1 as monotherapy whereas for the other patient HFS resolved upon S-1 dose reduction.

\section{Conclusions}

The SCOOP study provides important information regarding the safety and tolerability of S-1 for patients with advanced esophagogastric adenocarcinoma treated in different European centers. Most patients received approximately $70 \%$ of the planned dose and S-1-based treatment had a manageable adverse-event profile in this setting. In particular, patients who have discontinued capecitabine or 
5-FU because of HFS and cardiovascular adverse events can be safely switched to S-1 regimens. This real-world data on treatment compliance and adverse events may be of value to physicians when choosing between available options for the treatment of patients with advanced esophagogastric cancer.

Acknowledgments The author expresses his appreciation for the important contribution of the physicians and their colleagues in the five countries that enrolled patients in this observatory and entered the data into the database. In particular, a special thanks to the following physicians who have included a significant number of patients: Prof. Michalis Karamouzis (National and Kapodistrian University of Athens, Athens, Greece), Dr. Anna Koumarianou (Attikon University Hospital, Athens, Greece), Dr. Gerasimos Aravantinos and Dr. Joseph Sgouros (Agioi Anargiroi Cancer Hospital, Athens, Greece), Dr. Gerardo Rosati (S. Carlo Hospital, Potenza, Italy), and Dr. Kyran Bulger (Midland Regional hospital, Tullamore, Ireland). In addition, the author acknowledges the contributions of Sandy Field, PhD and Wilko Coers, PhD (Meducom BV, Spierdijk, The Netherlands) in their thorough data analysis and preparation of this article.

\section{Compliance with Ethical Standards}

Funding Nordic Pharma provided financial assistance for the preparation of this article. The patient data collection was managed by an independent party Quanta-Medical (France), supported by Nordic Pharma.

Conflict of Interest Marino Venerito received honoraria from Merck Serono, Bayer Vital, and Sirtex and is a member of the advisory boards of Ipsen, Lilly, Nordic Pharma, BMS, MSD, and Amgen.

Ethics Approval The study was approved by the institutional review board and/or ethics committee at each participating center and was in accordance with the Helsinki Declaration of 1975, as revised in 1983.

Open Access This article is distributed under the terms of the Creative Commons Attribution-NonCommercial 4.0 International License (http://creativecommons.org/licenses/by-nc/4.0/), which permits any noncommercial use, distribution, and reproduction in any medium, provided you give appropriate credit to the original author(s) and the source, provide a link to the Creative Commons license, and indicate if changes were made.

\section{References}

1. Robert Koch Institute, Association of Population-Based Cancer Registries in Germany. Cancer in Germany 2009/2010. 2014.

2. Jin H, Pinheiro PS, Callahan KE, Altekruse SF. Examining the gastric cancer survival gap between Asians and whites in the United States. Gastric Cancer. 2017;20:573-82.

3. Cunningham D, Allum WH, Stenning SP, Thompson JN, Van de Velde CJ, Nicolson M, Scarffe JH, Lofts FJ, Falk SJ, Iveson TJ, Smith DB, Langley RE, Verma M, Weeden S, Chua YJ; MAGIC Trial Participants. Perioperative chemotherapy versus surgery alone for resectable gastroesophageal cancer. N Engl J Med. 2006;355:11-20.

4. Bose K, Franck C, Müller MN, Canbay A, Link A, Venerito M. Perioperative therapy of oesophagogastric adenocarcinoma: mainstay and future directions. Gastroenterol Res Pract. 2017;2017:5651903.

5. Waddell T, Verheij M, Allum W, Cunningham D, Cervantes A, Arnold D. Gastric cancer: ESMO-ESSO-ESTRO clinical practice guidelines for diagnosis, treatment and follow-up. Radiother Oncol. 2014;110:189-94.

6. National Comprehensive Cancer Network (NCCN). Clinical practice guidelines in oncology: gastric cancer. Version 2. 2018. https://www.nccn.org/. Accessed 28 Nov 2018.

7. European Medicines Agency. Teysuno ${ }^{\circledR}(\mathrm{S}-1)$ : summary of product characteristics. https://www.ema.europa.eu/documents/ product-information/teysuno-epar-product-information_en.pdf. Accessed 28 Nov 2018.

8. Ajani JA, Rodriguez W, Bodoky G, Moiseyenko V, Lichinitser M, Gorbunova V, Vynnychenko I, Garin A, Lang I, Falcon S. Multicenter phase III comparison of cisplatin/S-1 with cisplatin/ Infusional fluorouracil in advanced gastric or gastroesophageal adenocarcinoma study. J Clin Oncol. 2010;28:1547-53.

9. Ter Veer E, Ngai LL, Valkenhoef GV, Mohammad NH, Anderegg MCJ, van Oijen MGH, van Laarhoven HWM. Capecitabine, 5-fluorouracil and S-1 based regimens for previously untreated advanced oesophagogastric cancer: a network metaanalysis. Sci Rep. 2017;7:7142.

10. Koizumi W, Tanabe S, Saigenji K, Ohtsu A, Boku N, Nagashima F, Shirao K, Matsumura Y, Gotoh M. Phase I/II study of S-1 combined with cisplatin in patients with advanced gastric cancer. Br J Cancer. 2003;89:2207-12.

11. Ajani JA, Faust J, Ikeda K, Yao JC, Anbe H, Carr KL, Houghton M, Urrea P. Phase I pharmacokinetic study of S-1 plus cisplatin in patients with advanced gastric carcinoma. J Clin Oncol. 2005;23:6957-65.

12. Kwakman JJM, Simkens LHJ, van Rooijen JM, van de Wouw AJ, Ten Tije AJ, Creemers GJM, Hendriks MP, Los M, van Alphen RJ, Polee MB, Muller EW, van der Velden AMT, van Voorthuizen T, Koopman M, Mol L, van Werkhoven E, Punt CJA. Randomized phase III trial of S-1 versus capecitabine in the first-line treatment of metastatic colorectal cancer: SALTO study by the Dutch Colorectal Cancer Group. Ann Oncol. 2017;28:1288-93.

13. Kwakman JJM, Baars A, Boot H, Pruijt JFM, Winther SB, Pfeiffer P, Punt CJA. Tolerability of the oral fluoropyrimidine S-1 after hand-foot syndrome-related discontinuation of capecitabine in Western cancer patients. Acta Oncol. 2017;56:1023-6.

14. Kwakman JJ, Simkens LH, Mol L, Kok WE, Koopman M, Punt CJ. Incidence of capecitabine-related cardiotoxicity in different treatment schedules of metastatic colorectal cancer: a retrospective analysis of the CAIRO studies of the Dutch Colorectal Cancer Group. Eur J Cancer. 2017;76:93-9.

15. Kwakman JJM, Baars A, van Zweeden AA, de Mol P, Koopman M, Kok WEM, Punt CJA. Case series of patients treated with the oral fluoropyrimidine S-1 after capecitabine-induced coronary artery vasospasm. Eur J Cancer. 2017;81:130-4.

16. Franck C, Malfertheiner P, Venerito M. Safe administration of S-1 after 5-fluorouracil-induced cardiotoxicity in a patient with colorectal cancer. BMJ Case Rep. 2017;2017. pii: bcr-2016-219162.

17. Common Terminology Criteria for Adverse Events, Version 3.0. http://ctep.cancer.gov/protocolDevelopment/electronic_applicatio ns/docs/ctcaev3.pdf. Accessed 28 Nov 2018.

18. Lauren P. The two histological main types of gastric carcinoma: diffuse and so-called intestinal-type carcinoma. An attempt at a histo-clinical classification. Acta Pathol Microbiol Scand. 1965;64:31-49.

19. Conroy T, Hammel P, Hebbar M, Ben Abdelghani M, Wei AC, Raoul JL, Choné L, Francois E, Artru P, Biagi JJ, Lecomte T, Assenat E, Faroux R, Ychou M, Volet J, Sauvanet A, Breysacher G, Di Fiore F, Cripps C, Kavan P, Texereau P, Bouhier-Leporrier 
K, Khemissa-Akouz F, Legoux JL, Juzyna B, Gourgou S, O'Callaghan CJ, Jouffroy-Zeller C, Rat P, Malka D, Castan F, Bachet JB; Canadian Cancer Trials Group and the UnicancerGI-PRODIGE Group. FOLFIRINOX or gemcitabine as adjuvant therapy for pancreatic cancer. N Engl J Med. 2018;379:2395-406.

20. Reiss KA, Yu S, Mamtani R, Mehta R, D'Addeo K, Wileyto EP, Taddei TH, Kaplan DE. Starting dose of sorafenib for the treatment of hepatocellular carcinoma: a retrospective, multi-institutional study. J Clin Oncol. 2017;35:3575-81.

21. Leicher LW, de Graaf JC, Coers W, Tascilar M, de Groot JW. Tolerability of capecitabine monotherapy in metastatic colorectal cancer: a real-world study. Drugs R D. 2017;17:117-24.

22. Franck C, Canbay A, Malfertheiner P, Venerito M. Neoadjuvant treatment with cisplatin and S-1 in elderly patients with oesophagogastric adenocarcinoma and locoregional disease: two case reports and review of the literature. Mol Clin Oncol. 2017:7:1069-72.

23. Zaanan A, Bouché O, Benhaim L, Buecher B, Chapelle N, Dubreuil O, Fares N, Granger V, Lefort C, Gagniere J, Meilleroux J, Baumann AS, Vendrely V, Ducreux M, Michel P; Thésaurus National de Cancérologie Digestive (TNCD). Gastric cancer: French intergroup clinical practice guidelines for diagnosis, treatments and follow-up (SNFGE, FFCD, GERCOR, UNICANCER, SFCD, SFED, SFRO). Dig Liver Dis. 2018;50:768-79.

24. Smyth EC, Verheij M, Allum W, Cunningham D, Cervantes A, Arnold D; ESMO Guidelines Committee. Gastric cancer: ESMO clinical practice guidelines for diagnosis, treatment and follow-up. Ann Oncol. 2016;27(Suppl. 5):v38-49.

25. Lokich J. What is the "best" platinum: cisplatin, carboplatin, or oxaliplatin? Cancer Invest. 2001;19:756-60. 